\title{
Transcription fluctuation effects on biochemical oscillations
}

Ryota Nishino, Takahiro Sakaue, Hiizu Nakanishi

\section{Foot notes}

1. In their work[12], the reaction rates for the gene processes $a_{i}$ and $d_{i}(i=1 \sim 4)$ are taken to be proportional to $\Omega$ as shown in Table 2 of Supporting information for ref.[12].

2. Ref.[12] examined the case of the sequential $\mathrm{P}_{\mathrm{N}}$ bindings, but there are other possible decompositions of the process such as that some dimers or oligomers bind to the gene after being formed in the nucleus.

3. Ref.[12] used $\Omega$ as a scaling parameter in the places where we use $1 / \tau$. 